\title{
Application of a lanthanide tag for evaluation of conformational states of a multidomain protein
}

\author{
T. Saio ${ }^{1}$, H. Nakagawa ${ }^{2}$, S. Hiramatsu' ${ }^{3}$ M. Asada ${ }^{4}$, H. Kawamukai ${ }^{1,3}$, T. Nakamura ${ }^{4}$, K. Ishimori ${ }^{3,5}$ \\ ${ }^{1}$ Institute of Advanced Medical Sciences, Tokushima University, Tokushima, Japan, ${ }^{2}$ Materials Sciences Research Center, Japan \\ Atomic Energy Agency, Tokai, Japan, ${ }^{3}$ Graduate School of Chemical Sciences and Engineering, Hokkaido University, Sapporo, \\ Japan, ${ }^{4}$ Instrument Center, Institute for Molecular Science, Okazaki, Japan, ${ }^{5}$ Department of Chemistry, Faculty of Science, Hokkaido \\ University, Sapporo, Japan. \\ saio@tokushima-u.ac.
}

Despite their importance in function, the conformational states and changes of proteins are often poorly understood mainly because of the lack of an efficient tool. MurD, a 47-kDa three-domain protein enzyme responsible for peptidoglycan biosynthesis, is one of those proteins whose conformational states and changes during its catalytic cycle are not well understood. The previous crystallographic studies have identified two major conformational states of MurD, open and closed conformations, in which the domain 3 has distinct orientations with respect to the other two domains. The conformational difference between the two crystal structures suggested that MurD can undergo drastic conformational changes in solution. However, the details about the conformational states and changes of MurD in solution coupled with the binding with the ligands or the inhibitors remained to be elucidated.

In our study, we exploited multiple biophysical methods including nuclear magnetic resonance (NMR), electron paramagnetic resonance (EPR), small-angle X-ray scattering (SAXS), and molecular dynamics (MD) simulation to demonstrate evaluation of the conformational states and distribution of MurD. We exploited paramagnetic lanthanide ions that can be attached to the specific position(s) on the protein by the use of the lanthanide tags [1]. In NMR, the effects of the paramagnetic lanthanide ions are observed as pseudo-contact shifts (PCSs) that can provide long-range $(<\sim 40 \AA)$ distance and angular information of each of the observed nuclei in the protein [1]. The lanthanide ion was fixed on the domain 2 of MurD and PCSs were observed from the resonances derived from the domain 3. Analysis of PCSs achieved estimation of conformational states of MurD in solution and detection of the conformational changes of MurD induced by its ligands and inhibitors [2]. The paramagnetic lanthanide ions, especially gadolinium ions, can be exploited by EPR and double electron-electron resonance (DEER) measurement that provides inter-gadolinium distance and population (distance distribution) [1]. The distance distributions obtained from DEER measurement were consistent with the information derived from PCS-NMR, SAXS, and MD simulation.

Our study highlights several biophysical methods to investigate the overall conformational states of a multi-domain protein. The integrated use of these methods can be an efficient strategy to evaluate the conformational states and distribution of proteins in solution.

[1] Saio, T., Ishimori, K. (2020) Biochim. Biophys. Acta. Gen. Subj. 1864.

[2] Saio, T., Ogura, K., Kumeta, H., Kobashigawa, Y., Shimizu, K., Yokochi, M., Kodama, K., Yamaguchi, H., Tsujishita, H., Inagaki, F. (2015) Sci. Rep. 5, 16685.

Keywords: Multi-domain protein, integrative structural biology, lanthanide ion 